\title{
Development of dual-curing type experimental composite resin cement for orthodontic bonding -Effect of additional amount of accelerators on the mechanical properties-
}

\author{
Jun LI, Isao SHIBUYA, Ichiko TESHIMA, Kimiya NEMOTO and Norihiro NISHIYAMA \\ Department of Dental Biomaterials, Nihon University School of Dentistry at Matsudo, 870-1 Sakaecho, Nishi 2, Matsudo, Chiba 271-8587, Japan \\ Corresponding author, Norihiro NISHIYAMA; E-mail: address: nishiyama.norihiro@nihon-u.ac.jp
}

\begin{abstract}
In this study, a dual-curing type composite resin cement that included a photo-initiator and two accelerators was designed. In particular, special emphasis was made on addressing questions on the effects from different amounts of additional accelerators on the flexural strength of the designed experimental composite resin cement, as well as on the tensile bond strength of the bracket bonded onto the enamel surface by the experimental composite resin cement.

When 0.25 mass $\%$ of the $p$-tolydiethanolamine and sodium $p$-toluenesulfinate were added, the maximum flexural strength was obtained for the chemical-cured and dual-cured experimental composite resin cement. The dual-cured experimental composite resin cement's flexural strength value was in the mid-range of the values exhibited from the commercial resin cements. However, the dualcured experimental composite resin cement exhibited noticeably high tensile bond strength when compared with the results obtained with the commercial resin cements.
\end{abstract}

Keywords: Resin cement for bracket bonding, Flexural strength, Tensile bond strength of bracket

Received Sep 1, 2008: Accepted Dec 22, 2008

\section{INTRODUCTION}

The introduction of the acid-etched technique to orthodontic bonding by Buonocore ${ }^{1)}$, has facilitated the development of many different types of bonding systems $^{2,3)}$ and numerous types of resin cements ${ }^{4-6)}$ that are critical in the correct application of orthodontic forces.

In clinical practice, most orthodontists will activate the orthodontic appliances in the mouth from 15 to 30 minutes after using resin cement to bond the bracket onto the enamel surface. The initial bond strength of the bracket to the enamel surface is, therefore, very important in ensuring the successful application of the direct bonding orthodontic technique.

Currently, a wide variety of orthodontic resin cements are commercially available. The orthodontic resin cements are classified into one of three resin cement types of: 1) chemical-curing, 2) light-curing and 3) dual-curing (combination of chemical and light curing), resin cements. The chemical-curing type 4methacryloyloxyethyl trimellitate anhydride (4-META)/ methyl methacrylate (MMA) resin cement, also known as Super Bond, is widely used by orthodontists since the Super Bond provides noticeably high bond strength for bonding the bracket onto the enamel surface ${ }^{7,8}$. However, in practice, the clinician must wait approximately 4-8 minutes for the Super Bond to harden before proceeding with the orthodontic treatment.

In order to directly control the setting time of the resin cement and to obtain high bond strength of the bracket immediately after bonding, clinicians utilize a light-curing type resin cement to bond the bracket to the enamel surface ${ }^{9-11)}$. However, when the metal bracket is placed onto the enamel surface, the metal bracket inhibits the transmittance of the visible light that is necessary for initiating the polymerizing reaction of the light-curing type resin cement that exists under the bracket. Thus, drawbacks in the application of light-curing resin cements are: l) amount of time that is required to cure the resin cement under the metal bracket, and 2) regardless of the curing time that is used, the clinician may never be totally assured of the complete polymerization of the resin under the bracket $^{12)}$. Incomplete polymerized areas within the adhesive layer of the resin cement may allow for the diffusion of water that may impair the bond strength in these areas and thus compromise the long-term effectiveness of the adhesive resin cement.

So as to overcome these drawbacks, as well as, to directly control the setting time of the resin cement and to improve the polymerization conversion of the resin cement that exists under the metal bracket, a dual-curing type composite resin cement, designed with additional capabilities, e.g. self-adhesiveness and fluoride release, was developed by adding 4methacryloyloxy ethyl trimellitic acid (4-MET) ${ }^{13-15)}$, Penta(methacryloxy-ethyl-oxy)-cyclophosphazene monofluoride (PEM-F) ${ }^{16)}$ to the base monomer of the experimental resin cement.

In this study, a dual-curing type composite resin cement that consisted of two types of pastes, a photoinitiator or accelerators for a photo and chemical polymerizations, was designed. In particular, special emphasis was placed on addressing questions on the effects from different amounts of additional photo and chemical accelerators on the flexural strength of the designed experimental composite resin cement, and on the tensile bond strength of the bracket bonded onto 
the enamel surface by the experimental composite resin cement.

\section{MATERIALS AND METHODS}

The designed dual-curing type composite resin cement consisted of two types of pastes. The components and compositions of the base monomer $\mathrm{A}$ for paste $\mathrm{A}$ and base monomer B for paste B are shown in Table 1 . There, $p$-tolyldiethanolamine ( $p$-TDEA; Tokyo Chemical Industry Co., Tokyo, Japan) and sodium $p$ toluenesulfinate ( $p$-TSNa; Tokyo Chemical Industry Co., Tokyo, Japan) were used as accelerators for photo or chemical polymerization. The amount of additional accelerators, $p$-TDEA and $p$-TSNa to base monomer B were $0.125,0.25,0.5,1$ and 2.5 mass\%, respectively. Furthermore, 0.5 mass\% of camphorquinone (CQ; Wako, Osaka, Japan), as a photo initiator, was dissolved in base monomer A.

In addition, a base monomer $\mathrm{A}$ without $\mathrm{CQ}$ for chemical-curing and a base monomer $\mathrm{B}$ with 0.25 mass $\%$ of $p$-TDEA only for light-curing were also prepared.

During the preparation of the experiment composite resin cement, colloidal silica (Aerosil 130, Aerosil Nippon Co, Tokyo, Japan) was used as filler. The surface of the colloidal silica was silanated by 6 mass $\%$ of $\gamma$-methacryloxypropyl trimethoxysilane. After which, $10 \mathrm{~g}$ or $8 \mathrm{~g}$ of silanated colloidal silica was filled into $10 \mathrm{~g}$ of base monomer $\mathrm{A}$ and base monomer $\mathrm{B}$, respectively.

A light-curing type composite resin cement (Kurasper ${ }^{\mathbb{B}}$ F, KF; Kuraray Medical Inc., Osaka, Japan) and a chemical-curing type 4-META/MMA resin cement (Super Bond, SB; Sun Medical, Shiga, Japan) were used as a control.

Measurement of gelation time of base monomer mixture Equal amounts of base monomer A without CQ and base monomer B with different amounts of accelerators, $p$-TDEA and $p$-TSNa, were weighted and then mixed at room temperature by using a plastic spatula on a mixing paper for 20 seconds. After mixing, the base monomer mixture was collected in a mixing dish. The surface of the mixed monomer was then covered with poly vinylidene chloride (Saran Wrap ${ }^{\circledR}$, Asahi Kasei Corp., Tokyo, Japan). Thereafter, the surface of the mixed base monomer was probed by using an explorer. The gelation times of the base monomer mixtures were measured while varying the amount of additional accelerators. Measurements of the gelation time were repeated six times for each experimental group inside of a dark room.

\section{ESR measurement}

The base monomer A without $\mathrm{CQ}$ and base monomer $\mathrm{B}$ with accelerators, $p$-TDEA and $p$-TSNa were mixed in a 1:1 ratio for 20 seconds. There, the amount of additional accelerators was 0.25 mass $\%$, respectively. Next, the base monomer mixture was inserted into a silica tube for electron spin resonance (ESR) measurement. The ESR spectra of the chemical-cured base monomer mixture during hardening were then observed as a function of time by using an ESR spectrum apparatus (JES FA 300, JEOL, Tokyo, Japan). The measurement conditions were as follows: microwave power of $8 \mathrm{mM}$; magnetic field of $335.4 \pm 5$ $\mathrm{mT}$; sweep time of 2 minutes; modulation frequency of $100 \mathrm{kHz}$; and time constant of 0.03 seconds.

In addition, when the mixture of the base monomer A with 0.5 mass\% of $\mathrm{CQ}$ and base monomer $\mathrm{B}$ with 0.25 mass $\%$ of the accelerators was dual-cured through visible-light irradiation by a light curing unit (XL3000, 3M-ESPE, Grafenau, Germany), the ESR spectra of the base monomer mixture were observed as a function of time after visible-light irradiation. There, the mixing of the base monomers $\mathrm{A}$ and $\mathrm{B}$, and the measurement of the ESR spectrum of each sample were conducted. The

Table 1 Components and compositions of base monomer A and B for the experimental composite resin cement

\begin{tabular}{clr}
\hline Base monomer A & \multicolumn{1}{c}{ Manufacture } & mass\% \\
\hline Bis-GMA & Polysciences Inc., Warrington, USA & 6.7 \\
HEMA & Polysciences Inc., Warrington, USA & 26.7 \\
4 -MET & Dentsply-Sankin, Tokyo, Japan & 20.0 \\
CB-1 & Shin-Nakamura Chemical, Wakayama, Japan & 13.3 \\
PEM-F & Fushimi Pharmaceutical, Marugame, Japan & 64.3 \\
Base monomer B & & 35.7 \\
Bis-GMA & Polysciences Inc., Warrington, USA & \\
TEGDMA & Wako Pure Chemical Inc, Osaka, Japan & \\
\hline
\end{tabular}

Bis-GMA: bisphenol A glycidyl dimethacrylate; HEMA: 2-hydroxyethyl methacrylate; 4-MET: 4-methacryloyloxyethyl dihydrogen trimellitate; CB-1: $\beta$-methacryloyloxyethyl hydrogen phthalate; PEM-F: Penta(methacryloxy-ethyl-oxy)cyclophosphazene mono-fluoride; TEGDMA: triethylene glycol dimethacrylate 
ESR observations of the dual-cured and chemical-cured base monomer mixtures were performed two times inside of a dark room.

\section{Measurement of flexural strength}

The experimental composite resin cement consisting of paste A with 0.5 mass $\%$ of $\mathrm{CQ}$ and paste $\mathrm{B}$ with different amounts of accelerator, $p$-TDEA and $p$-TSNa, were mixed in a 1:1 ratio for 20 seconds. Each paste mixture was then filled into a stainless steel split mold that had been glued onto a glass slide. The split mold was then used to produce a specimen bar, where the experimental composite resin cement had hardened with a width of $4.2 \mathrm{~mm}$, a height of $2.1 \mathrm{~mm}$ and a length of $35 \mathrm{~mm}$. A transparent thin film was placed on the top surface of the mixture. Visible-light was then irradiated on the mixture from the transparent film side for 30 seconds, and then irradiated on the mixture from the glass slide side for 30 seconds, respectively, by using a light-curing unit ( $\alpha$-light II, Morita, Tokyo, Japan). After removing the specimens from the split mold, they were immersed in water at $37^{\circ} \mathrm{C}$ for 1 day. After which, the specimens were polished with a sequence of 600-grit and 1000-grit carbide papers under a stream of water. The width and height of the hardened specimen bars were fixed to $4.0 \mathrm{~mm}$ and 2.0 $\mathrm{mm}$, respectively. The specimens were then placed on a three-point bending fixture mounted on a universal testing machine (TG-5KN, Minebea, Kanagawa, Japan). There, the span distance for the three-point bending fixture was $10 \mathrm{~mm}$ and a crosshead was placed at the center of the bending fixture. A load was applied to the specimen bar under a crosshead speed of $1 \mathrm{~mm} / \mathrm{min}$. Concurrently, the load-deflection curve was recorded on the computer. Using the load-deflection curve, the flexural strength was derived from the maximum load and the elastic modulus was derived from the deflection when the load was $0.4 \mathrm{kN}$. The maximum deflection was determined from the load-deflection curve where the maximum load was detected.

Furthermore, the flexural strength of the chemicalcured experimental composite resin cement, consisting of paste $\mathrm{A}$ without $\mathrm{CQ}$ and paste $\mathrm{B}$ with different amounts of accelerators, $p$-TDEA and $p$-TSNa, was measured as per the previously discussed methods. The flexural strength of the light-cured experimental composite resin cement consisting of paste A with 0.5 mass \% of CQ and paste B with 0.25 mass\% of $p$-TDEA only, was also determined.

In addition, the flexural strengths of two commercial resin cements, KF and SB were measured as per the previously discussed methods, after the specimens were prepared, as a control.

The number of specimens for each experimental group was seven. The mean values of flexural strength, elastic modulus, maximum deflection and their standard deviation (SD) were calculated for each experimental group. The results were analyzed by oneway analysis of variance (ANOVA) and Scheffé's multiple comparison tests.
Preparation of the specimens for tensile bond strength test

Fresh, intact, anterior human teeth that were immediately stored in water at $4{ }^{\circ} \mathrm{C}$ after extraction were used for the tensile bond strength test. After cutting the root, the labial enamel surface of each anterior tooth was cleaned, rinsed, and dried according to generally accepted procedures. The labial enamel surface was then etched with an etching gel (Xeno Ortho, Dentsply-Sankin, Tokyo, Japan) for 30 seconds, rinsed for 20 seconds, and then dried for 30 seconds.

A metal bracket (Standard number 105-1100, Dentsply-Sankin, Tokyo, Japan) was bonded to the conditioned enamel by applying the dual-curing type experimental composite resin cement. There, the amount of additional accelerators, $p$-TDEA and $p$ TSNa, was 0.25 mass\%. Next, visible light was irradiated at a $45^{\circ}$ angle to the mesial-gingival corner of the bracket for 5 seconds and then at a $45^{\circ}$ angle to the distal-occlusal corner for another 5 seconds by using a light curing unit.

The bonded specimens with a metal bracket adhered to the labial enamel surface by the chemicalcured experimental composite resin cement or lightcured experimental composite resin cement with visible-light irradiation were also prepared using the same methods as previously described. There, when the bracket was bonded to the enamel surface by the lightcuring type experimental composite resin cement, visible light was irradiated at a $45^{\circ}$ angle to the mesialgingival corner of the bracket for 30 seconds and then at a $45^{\circ}$ angle to the distal-occlusal corner for another 30 seconds.

Furthermore, commercial resin cements, KF or SB were applied to bond the metal bracket to the labial enamel surface, as per the respective resin cements instructions. The bonded specimens were stored in water at $37^{\circ} \mathrm{C}$ for 1 day.

\section{Measurement of tensile bond strength}

After being immersed in water at $37^{\circ} \mathrm{C}$ for 1 day, the bonded specimens were molded with a self-curing pour resin (Shofu Inc, Kyoto, Japan). Next, an orthodontic wire $(0.457 \times 0.558 \mathrm{~mm}$; RMO Inc., Denver, USA) was fastened to the metal bracket by using a ligature wire (0.305 mm; RMO Inc.). The molded specimens were then mounted on a universal testing machine. After hooking the orthodontic wire with a stainless steel device, the orthodontic bracket was vertically pulled against the enamel surface under a crosshead speed of $1 \mathrm{~mm} / \mathrm{min}$. The tensile bond strength of the orthodontic bracket to the enamel surface was thereby measured.

The number of specimens for each experimental group was 7 . The mean value of tensile bond strength and its standard deviation (SD) were calculated for each experimental group. The results were analyzed by one-way analysis of variance (ANOVA) and Scheffé's multiple comparison tests. 


\section{RESULTS}

Gelation time of the base monomer mixtures for chemical-cured experimental composite resin cement

Figure 1 shows the effects from the amounts of additional accelerators, $p$-TDEA and $p$-TSNa, to the base monomer $\mathrm{B}$ on the gelation time when the mixture of base monomers $\mathrm{A}$ and $\mathrm{B}$ was chemical-cured. In this case, the base monomer A without $\mathrm{CQ}$ was utilized.

When the amount of additional accelerators to the base monomer B was increased from 0.125 to 2.5 mass\%, the gelation time was reduced from 554.2 to 172.0 seconds.

Figures 2 and 3 show the ESR spectra of the mixture of base monomers $\mathrm{A}$ and $\mathrm{B}$ when the mixture was chemical-cured or dual-cured. In both case, the amount of accelerators, $p$-TDEA and $p$-TSNa, added to base monomer B was 0.25 mass $\%$.

When the mixture of base monomer A without CQ and base monomer $\mathrm{B}$ with the accelerators was chemical-cured, no ESR signals were detected in the ESR spectra, as shown in Fig. 2. In addition, it took 491 seconds for the base monomer mixture to harden into a gel.

In contrast, when the mixture of the base monomer A with 0.5 mass\% of $\mathrm{CQ}$ and base monomer $\mathrm{B}$ with the accelerators was dual-cured through visible-light irradiation, three ESR signals were detected in the

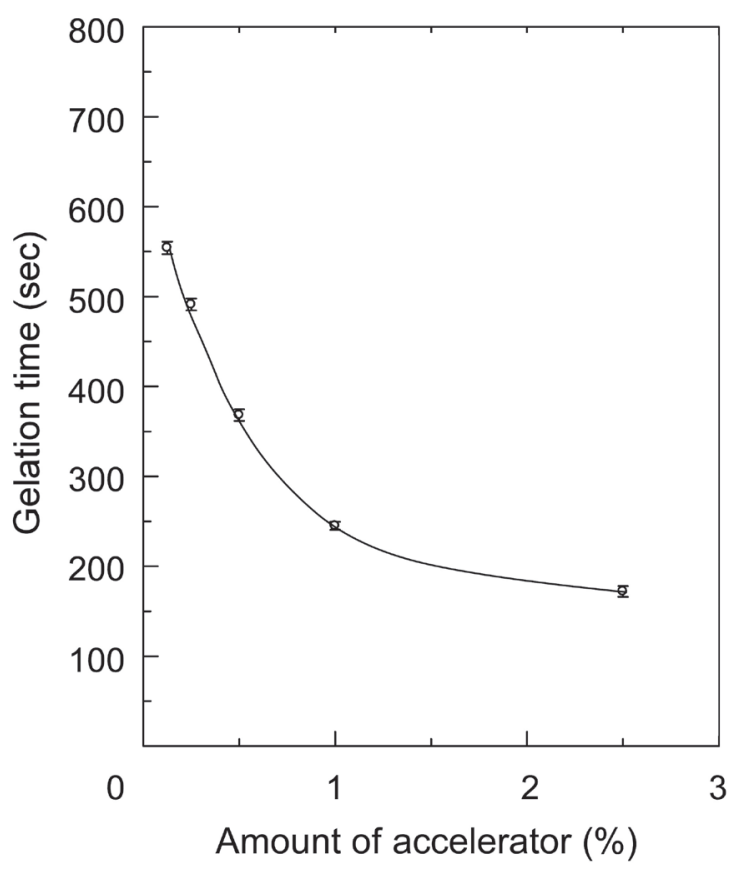

Fig. 1 Effects from different additional amounts of accelerator on the gelation time of the mixture of the base monomer $\mathrm{A}$ and $\mathrm{B}$ for the chemicalcuring type experimental resin cements. The CQ was not added to the base monomer A. The error bar shows SD.
ESR spectra, as shown in Fig. 3. The ESR signals were assigned to the carbon radical, since the g-value of the developed ESR signal was 2.0009.

Flexural strength of experimental composite resin cement

Figure 4 shows the effects from different amounts of additional accelerators, $p$-TDEA and $p$-TSNa, on the flexural strength of the experimental composite resin cements. The white circles indicate the flexural strength when the experimental composite resin cement, comprised of paste A with 0.5 mass $\%$ of $\mathrm{CQ}$ and paste B with accelerators, was dual-cured through visible-light irradiation. The white squares show the flexural strength when the experimental composite resin cement, comprised of paste $\mathrm{A}$ without $\mathrm{CQ}$ and paste B with accelerators, was chemical-cured.

When the amount of additional accelerators was $0.25 \mathrm{mass} \%$, the chemical-curing type experimental composite resin cement exhibited a maximum flexural strength of $65.3 \mathrm{MPa}$. However, further increases in the amount of additional accelerators resulted in decreases in the flexural strength.

Conversely, when the experimental composite resin cement was dual-cured, the mean flexural strength significantly increased to above 90.0 MPa. For all additional amounts of accelerators, the dual-cured experimental composite resin cement provided noticeably higher flexural strength than that of the chemical-cured experimental composite resin cement $(p<0.05)$. However, the effects from the amount of additional accelerators on the flexural strength resulted

Mno

Mno
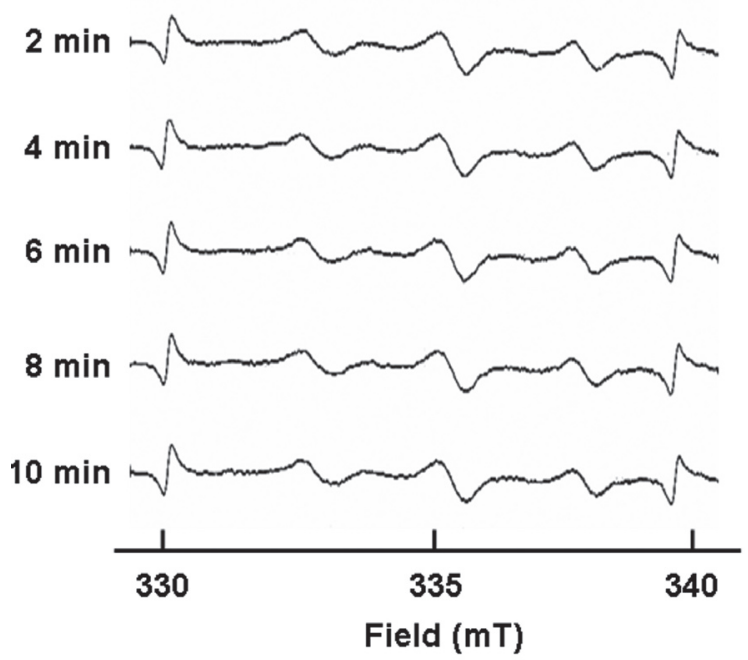

Fig. 2 ESR spectra of chemical-curing type base monomer mixture as a function of curing time. The amount of additional $p$-TDEA and $p$-TSNa used for base monomer B was 0.25 mass\%. The CQ was not added to the base monomer A. 
in almost the same as that of the chemical-cured experimental composite resin cement. The addition of 0.25 mass $\%$ of accelerators also exhibited a maximum flexural strength of 114.6 MPa.

Comparison of the mechanical properties and tensile bond strengths of the experimental composite resin cement with those of the commercial resin cements

Table 2 shows the flexural strengths, elastic moduli and maximum deflections of the experimental composite resin cements and of the commercial resin cements, as well as, the tensile bond strengths of the

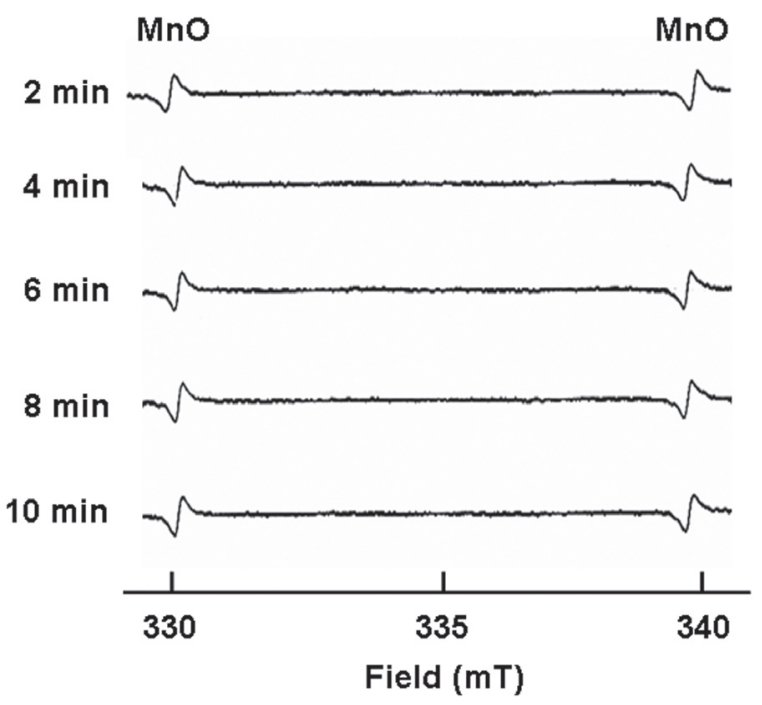

Fig. 3 ESR spectra of dual-curing type base monomer mixture as a function of curing time. The amount of additional $p$-TDEA and $p$-TSNa used for base monomer B was 0.25 mass\%. The amount of additional $\mathrm{CQ}$ used for base monomer $\mathrm{A}$ was 0.5 mass\%. bracket bonded to the enamel by the experimental composite resin cements or by the commercial resin cements. There, when the experimental composite resin cement was dual-cured, paste A with 0.5 mass\% of CQ and paste $\mathrm{B}$ with 0.25 mass $\%$ of $p$-TDEA and $p$-TSNa were utilized. In contrast, when the experimental composite resin cement was chemical-cured, paste $\mathrm{A}$ without $\mathrm{CQ}$ and paste $\mathrm{B}$ with 0.25 mass $\%$ of $p$-TDEA

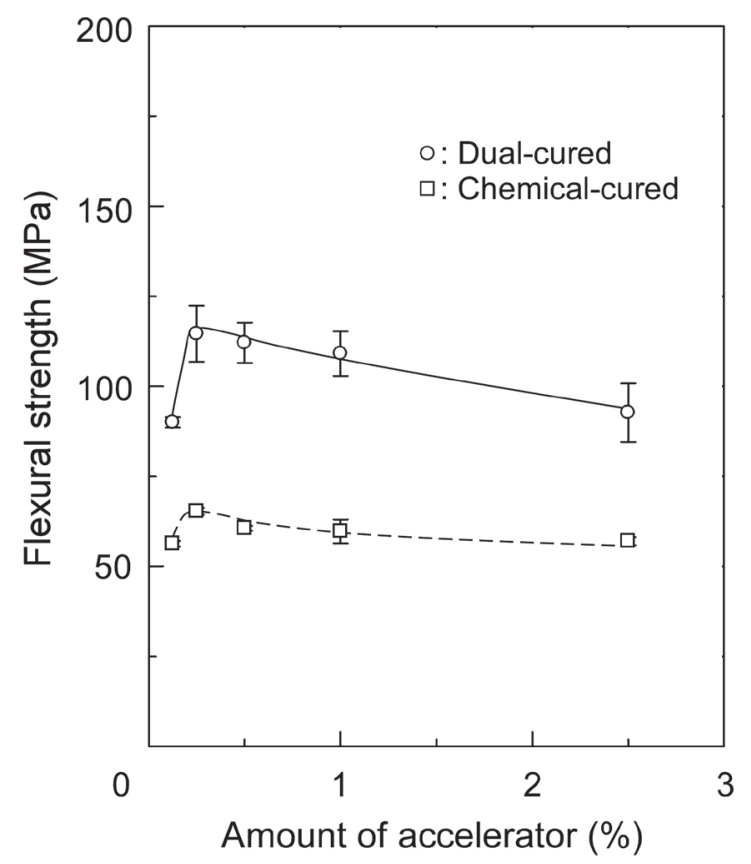

Fig. 4 Effects from different additional amounts of accelerators to base monomer $\mathrm{B}$ on the flexural strength of the experimental composite resin cements. The error bar shows SD.

Table 2 The flexural strengths, maximum deflections and elastic moduli of the experimental composite resin cements and the commercial resin cements, as well as the tensile bond strengths of the bracket bonded to the enamel by the experimental composite resin cements and the commercial resin cements

\begin{tabular}{lcccc}
\hline \multicolumn{1}{c}{ Resin cement } & $\begin{array}{c}\text { Flexural strength } \\
(\mathrm{MPa})\end{array}$ & $\begin{array}{c}\text { Deflection } \\
(\mathrm{mm})\end{array}$ & $\begin{array}{c}\text { Elastic } \\
(\mathrm{GPa})\end{array}$ & $\begin{array}{c}\text { Tensile bond strength } \\
(\mathrm{MPa})\end{array}$ \\
\hline Experimental resin cement & & & & \\
$\quad$ Dual-cure & $114.6(7.8)^{\mathrm{A}}$ & $0.20(0.03)^{\mathrm{A}}$ & $4.7(0.3)^{\mathrm{A}}$ & $7.5(0.8)^{\mathrm{A}}$ \\
$\quad$ Chemical-cure & $65.3(1.0)^{\mathrm{B}}$ & $0.31(0.04)^{\mathrm{B}}$ & $2.1(0.2)^{\mathrm{B}}$ & $4.4(1.2)^{\mathrm{B}}$ \\
$\quad$ Light-cure & $34.3(3.2)^{\mathrm{C}}$ & $0.29(0.05)^{\mathrm{B}}$ & $1.1(0.2)^{\mathrm{C}}$ & $4.1(0.3)^{\mathrm{B}}$ \\
Commercial resin cement & & & & \\
$\quad$ KF [Light-curing type] & $176.2(7.2)^{\mathrm{D}}$ & $0.19(0.01)^{\mathrm{A}}$ & $8.3(0.5)^{\mathrm{D}}$ & $6.5(2.1)^{\mathrm{A}}$ \\
SB [Chemical-curing type] & $88.0(1.8)^{\mathrm{E}}$ & $0.67(0.07)^{\mathrm{C}}$ & $1.7(0.1)^{\mathrm{E}}$ & $6.1(2.1)^{\mathrm{A}}$ \\
\hline
\end{tabular}

( ): SD

Within the same vertical column: median values with the different superscript letters (A-E) showing a statistical difference $(p<0.05)$.

KF: Kurasper ${ }^{\circledR}$ F (Kuraray Medical Inc., Osaka, Japan), SB: Super Bond (Sun Medical, Shiga, Japan) 
and $p$-TSNa were utilized. Conversely, when the experimental composite resin cement was light-cured, paste A with 0.5 mass\% of $\mathrm{CQ}$ and paste B with 0.25 mass $\%$ of $p$-TDEA only, were utilized.

The mean flexural strengths of the $\mathrm{SB}$ and $\mathrm{KF}$ were 88.0 and $176.2 \mathrm{MPa}$, respectively. The $\mathrm{KF}$ resin cement exhibited the highest flexural strength. The flexural strengths of the dual-cured, chemical-cured and light-cured experimental composite resin cements were 114.6, 65.3 and $34.3 \mathrm{MPa}$, respectively.

The mean elastic moduli of the SB and KF were 1.7 and $8.3 \mathrm{GPa}$, respectively. The $\mathrm{KF}$ exhibited the highest elastic modulus. The elastic moduli of the dualcured, chemical-cured and light-cured experimental composite resin cements were 4.7, 2.1 and $1.1 \mathrm{GPa}$, respectively.

The mean maximum deflections of the $\mathrm{SB}$ and $\mathrm{KF}$ were 0.67 and $0.19 \mathrm{~mm}$, respectively. The SB exhibited the highest maximum deflection. The maximum deflections of the dual-cured, chemical-cured and lightcured experimental composite resin cements were 0.20 , 0.31 and $0.29 \mathrm{~mm}$, respectively.

The mean tensile bond strengths of the metal bracket bonded to the enamel surface by the SB and $\mathrm{KF}$ were 6.1 and $6.5 \mathrm{MPa}$, respectively. The tensile bond strengths of the dual-cured, chemical-cured and light-cured experimental composite resin cements were 7.5, 4.4 and $4.1 \mathrm{MPa}$, respectively. The dual-curing type experimental composite resin cement exhibited the highest tensile bond strength.

\section{DISCUSION}

So as to better understand how the resin cement could better resist fracturing of the resin cement's adhesive layer that bonds the bracket to the enamel surface, it is important to examine the mechanical properties since the functional stresses, e.g. tensile, shear, torque, and peel, are exerted on the bonded bracket ${ }^{17,18}$.

In this study, in order to increase the bond strength of the metal bracket to the enamel surface by increasing the polymerization conversion of the resin cement under the bracket, a dual-curing type experimental composite resin cement was designed. The effects from different amounts of additional accelerators, $p$-TDEA and $p$-TSNa, on the flexural properties of the dual-curing type experimental composite resin cement were examined. The efficacy of this dual-curing type experimental composite resin cement, developed for direct bonding systems, was then discussed through a comparison with the mechanical properties of the commercial resin cements.

When the mixture of the base monomer A without $\mathrm{CQ}$ and base monomer $\mathrm{B}$ with accelerators, $p$-TDEA and $p$-TSNa was chemical-cured, the gelation time of the mixture became shorter as the amount of additional accelerators was increased. The gelation time of the mixture was strongly dependent on the amount of additional accelerators. In order to understand the chemical-curing mechanism of the base monomer mixture, ESR analyses were performed during hardening.

When the mixture of base monomer $\mathrm{A}$ with 0.5 mass\% of CQ and base monomer B with 0.25 mass\% of accelerators was dual-cured through visible-light irradiation, ESR signals, attributed to the carbon radical, were detected. The development of the carbon radical suggests that the dual-curing mechanism of the base monomer mixture is of a radical polymerization. This was possible since the irradiation of visible light resulted in the activation of the $\mathrm{CQ}$.

In contrast, when the mixture of the base monomer A without $\mathrm{CQ}$ and base monomer B with 0.25 mass\% of accelerators was chemical-cured, no ESR signal was observed. This result suggests that the chemical-curing mechanism of the mixture is not of a radical polymerization. The mixture may have been hardened by anionic polymerization, which could be initiated by the carboxylic acid in the 4-MET, and/or the CB-1 molecule, and the $p$-TSNa, as well as, by the $p$-TDEA ${ }^{19}$. In order to understand the chemical-curing mechanism of the base monomer mixture, further research should be conducted in the future.

Next, the experimental composite resin cements were prepared by mixing silanated colloidal silica to the base monomers A and B. The effects from different amounts of additional accelerators on the flexural strength of the experimental composite resin cement were then examined. When the amount of accelerators was 0.25 mass $\%$, the dual-cured and chemical-cured experimental composite resin cements exhibited the maximum flexural strengths. However, further increases in the amount of additional accelerators resulted in decreases in the mean flexural strength. These observed decreases in the flexural strength caused by increasing the amount of additional accelerators was caused by the $p$-TSNa and $p$-TDEA acting as a plasticizer, since both chemicals do not have a functional group in their molecules for polymerization. The flexural strength of the dual-cured experimental composite resin cement was in the mid-range of the values observed with the $\mathrm{KF}$ and $\mathrm{SB}$.

As shown in Table 2, we designed three experimental composite resin cements with different types of curing systems. It seemed that the mechanical properties of the experimental composite resin cement were strongly dependent on the type of curing system. The dual-curing type experimental composite resin cement exhibited higher mechanical properties, i.e. flexural strength and elastic modulus, than the chemical-curing and light-curing type experimental composite resins. The flexural strength, elastic modulus and maximum deflection of the experimental composite resin cement, in decreasing order were, dual-curing, chemical-curing and light-curing. The observed decreases in the mechanical properties of the chemicalcured and light-cured experimental resin cements, when compared to the dual-cured experimental resin cement, were probably due to decreases in the polymerization conversion of the base monomer. 
Further, the commercial composite resin cement, $\mathrm{KF}$, exhibited noticeable higher flexural strength and elastic modulus, when compared with the experimental composite resin cements. The observed higher flexural strength and elastic modulus of the KF than those of the experimental composite resin cements were due to differences in the amount of filler content. In fact, the amount of KF's filler content (78.1 mass\% as determined by the Ash method $\left.{ }^{20}\right)$ is greater than those of the experimental composite resin cements.

Wright and Powers ${ }^{21)}$ reported on the reliability requirements for tensile bond strength in clinical applications. They cited a requirement of a maximum force exerted on a bracket of 5.9 MPa. The dual-cured experimental composite resin cement and the commercial $\mathrm{KF}$ and SB met this requirement. However, the chemical-cured and light-cured experimental composite resin cements were not able to meet this requirement. Specifically, the light-cured experimental composite resin cement exhibited the lowest tensile bond strength. This drawback was due to 1) the mechanical properties of the light-cured experimental composite resin cement being lower than the other two experimental composite resin cements and 2) the lightcured experimental composite resin cement that existed under the metal bracket could not polymerize completely, since the metal bracket inhibited the transmittance of visible light. In contrast, the dualcuring type experimental composite resin cement provided higher tensile bond strength than that of the light-cured experimental composite resin cement. This result was possible since the dual-cured experimental composite resin cement that existed under the metal bracket could be chemical-cured, even when visible light was directly unable to irradiate the dual-cured experimental composite resin cement that existed under the metal bracket.

The dual-curing type experimental composite resin cement exhibited noticeably high tensile bond strength when compared with the result obtained with the KF, even though the flexural strength of the experimental composite resin cement was lower than that of the KF $(p<0.05)$. Keizer et al. ${ }^{17)}$ reported that the flexural strength of the resin cement was related to the tensile bond strength of the bracket to the enamel. However, we did not observe any correlation between the flexural strength and tensile bond strength when the mechanical properties of the dual-curing type were compared with those of the KF. This observed difference in behaviors was attributed to the degree of polymerization conversion of the resin cement that existed under the metal bracket. Consequently, the light-curing type $\mathrm{KF}$ provided noticeably low tensile bond strength when compared with the result obtained with the dual-cured experimental composite resin cement, since the degree of polymerization conversion of the light-cured KF that existed under the metal bracket was lower, due to insufficient amount of visible light reaching the $\mathrm{KF}$ to activate the photo polymerization of the $\mathrm{KF}$ under the bracket, when compared with that of the KF that was not hindered by the metal bracket that was directly irradiated by visible light.

The observed enhancement in the tensile bond strength of the metal bracket bonded by the dual-cured experimental composite resin cement than that of the light-curing type $\mathrm{KF}$ was probably due to the polymerization conversion of the dual-curing experimental composite resin cement that existed under the metal bracket being greater than that of the light-curing type KF. This was possible since the carboxylic acid in the 4-MET, and/or the CB-1 molecule, the $p$-TSNa, as well as, the $p$-TDEA initiated the anionic polymerization of the base monomer that existed under the metal bracket. These results clearly suggest that the designed dual-curing type experimental composite resin cement would be an advantageous material for use in direct bonding systems for the bonding of the metal bracket to the enamel surface.

In this study, so as to provide additional capabilities to the experimental composite resin cement, 4-MET and PEM-F were utilized in the base monomer $\mathrm{A}$ as part of the experimental composite resin cement. In the future, investigation of the selfadhesiveness and fluoride release of the designed experimental composite resin cement should be conducted.

\section{CONCLUSION}

Under the conditions of this study, the following conclusions were made:

1. The addition of 0.25 mass $\%$ of $p$-TSNa and $p$-TDEA to base monomer $\mathrm{B}$ provided maximum flexural strengths after the experimental composite resin cement was chemical-cured or dual-cured.

2. The dual-cured experimental composite resin cement provided noticeably high tensile bond strength when compared with the result obtained with the commercial light-curing type composite resin cement, $\mathrm{KF}$, even though the flexural strength of the experimental composite resin cement was lower than that of the KF.

\section{REFERENCES}

1) Buonocore MG. A simple method of increasing the adhesion of acrylic filling materials to enamel surface. J Dent Res 1955; 34: 849-853.

2) Mizrahi E. Orthodontic bands and directly bonded brackets: a review of clinical failure rate. J Dent 1983; 3: 231-236.

3) Linn BJ, Berzins DW, Dhuru VB, Bradley TG. A comparison of bond strength between direct- and indirect-bonding methods. Angle Orthod 2006; 76: 289-294.

4) Fricker JP. A 12-month clinical evaluation of a lightactivated glass polyalkenoate (ionomer) cement for the direct bonding of orthodontic brackets. Am J Orthod Dentofac Orthop 1994; 105: 502-505.

5) Eliades T, Eliades G, Brantley WA, Johnston WM. Polymerization efficiency of chemically cured and visible light-cured orthodontic adhesives: degree of cure. Am J 
Orthod Dentofac Orthop 1995; 108: 294-301.

6) Kasuya K, Miyazaki Y, Ogawa N, Maki K, Manabe A, Itoh K, Tani C, Hisamitsu H, Fujishima A, Miyazaki T. Efficacy of experimental dual-cure resin cement for orthodontic direct bond system. Orthod Waves 2006; 65: 107-111.

7) Guan G, Takano-Yamamoto $T$, Miyamoto M, Hattori $T$, Ishikawa K, Suzuki K. Shear bond strengths of orthodontic plastic brackets. Am J Orthod Dentofacial Orthop 2000; 117: 438-443.

8) Meguro D, Hayakawa T, Kasai K. Efficacy of using orthodontic adhesive resin in bonding and debonding characteristics of a calcium phosphate ceramic bracket. Orthod Waves 2006; 65: 148-154.

9) Greenlaw R, Way DC, Galil KA. An in vitro evaluation of a visible light-cured resin as an alternative to conventional resin bonding systems. Am J Orthod Dentofacial Orthop 1989; 96: 214-220.

10) Armas Galindo HR, Sadowsky PL, Vlachos C, Jacobson A, Wallace D. An in vivo comparison between a visible lightcured bonding system and a chemically cured bonding system. Am J Orthod Dentofacial Orthop 1998; 113: 271275.

11) Yamamoto A, Yoshida T, Tsubota $K$, Takamizawa $T$, Kurokawa H, Miyazaki M. Orthodontic bracket bonding: enamel bond strength vs time. Am J Orthod Dentofac Orthop 2006; 130: 435.e1-435.e6.

12) Smith RT, Shivapuja PK. The evaluation of dual cement resins in orthodontic bonding. Am J Orthod Dentofac
Orthop 1993; 103: 448-451.

13) Tanaka T, Nagata K, Takeyama M, Atsuta M, Nakabayashi N, Masuhara E. 4-META opaque resin--a new resin strongly adhesive to nickel-chromium alloy. J Dent Res 1981; 60: 1697-1706.

14) Nakabayashi N, Kojima K, Masuhara E. The promotion of adhesion by the infiltration of monomers into tooth substrates. J Biomed Mater Res 1982; 16: 265-273.

15) Matsumura $H$, Tanaka $T$, Atsuta M. Effect of acidic primers on bonding between stainless steel and auto-polymerizing methacrylic resins. J Dent 1997; 25: 285-290.

16) Anzai M, Kobayashi H, Yoshihashi K, Nakajima Y, Nishiyama M. Synthesis of fluoride releasing phosphazene monomers and their application to dental resin. J J Dent Mater 1997; 16: 90-100.

17) Keizer S, ten Cate JM, Arends J. Direct bonding of orthodontic brackets. Am J Orthod 1976; 69: 318-327.

18) McCarthy MF, Hondrum SO. Mechanical and bond strength properties of light-cured and chemically cured glass ionomer cements. Am J Orthod Dentofac Orthop 1994; 105: 135-141.

19) Tsuruta T. Anion polymerization, 1st ed, Kagakudoujin, Kyoto, 1973, pp. 7-24

20) Nakaso $K$ and Yoshino Y. Shinjikkenkagaku kouza 9, Bunseki Kagaku II, 3th ed, Maruzen, Tokyo, 1980, pp. 149167.

21) Wright WL, Powers JM. In vitro tensile bond strength of reconditioned brackets. Am J Orthod 1985; 87: 247-252. 